\title{
Time-dependent density-functional theory for ultrafast interband excitations
}

\author{
V. Turkowski* and C. A. Ullrich ${ }^{\dagger}$ \\ Department of Physics and Astronomy, University of Missouri, Columbia, Missouri 65211, USA \\ (Received 19 September 2007; revised manuscript received 30 November 2007; published 21 February 2008)
}

\begin{abstract}
We formulate a time-dependent density-functional theory (TDDFT) in terms of the density matrix to study ultrafast phenomena in semiconductor structures. A system of equations for the density-matrix components, which is equivalent to the time-dependent Kohn-Sham equation, is derived. From this, we obtain a TDDFT version of the semiconductor Bloch equations, where the electronic many-body effects are taken into account, in principle, exactly. As an example, we study the optical response of a three-dimensional two-band insulator to an external short-time pulsed laser field. We show that the optical absorption spectrum acquires excitonic features when the exchange-correlation potential contains a $1 / q^{2}$ Coulomb singularity. A qualitative comparison of the TDDFT optical absorption spectra with the corresponding results obtained within the Hartree-Fock approximation is made.
\end{abstract}

DOI: 10.1103/PhysRevB.77.075204

PACS number(s): 71.10.-w, 71.15.Mb, 71.45.Gm

\section{INTRODUCTION}

Due to the demands of modern electronics, semiconductor devices are becoming smaller and faster, which means that applied external fields cause strongly inhomogeneous and nonequilibrium processes in such systems. A powerful approach to study the dynamical properties of potentially useful materials and devices is to apply short (femto- or picosecond) electric field pulses and to measure the response of the system. Recent progress in ultrafast laser pulse experimental techniques allows one to study the physical processes in such systems with a very high precision (for a review see, e.g., Refs. 1 and 2). For example, with this technique, one can measure nonequilibrium energy and momentum distributions or the dynamics of excited states. Therefore, it is necessary to develop theoretical tools to describe these experiments, as well as to understand ultrafast processes in small semiconductor devices, in general.

The theoretical description of ultrafast phenomena triggered by short-pulse laser fields is a complicated problem due to several reasons. One of the most difficult tasks is to take into account many-particle correlation effects properly. An external pulsed field causes the following main effects in the system: (i) direct electron photoemission, (ii) inverse electron photoemission, and (iii) absorption processes. The first two phenomena can be described in terms of free quasiparticles, which makes the problem relatively simple. A proper description of optical absorption spectra is a much more complicated task due to quasiparticle correlation effects. In particular, external laser pulses can create excitons or coupled electron-hole pairs. The problem of correctly describing optical absorption spectra with excitonic features in the case of applied short-time laser pulses is one of the great challenges in condensed matter physics.

For small devices in the presence of a short-pulse field, typical time scales are shorter than the Coulomb scattering time (see, for example, Ref. 3), which means that one cannot treat the Coulomb interaction effects by using a simple Boltzmann equation approach, where all the Coulomb effects are "hidden" in a scattering time parameter, $\tau$. Similarly, the semiconductor Bloch equation (SBE) approach, ${ }^{4}$ based on the Hartree-Fock (HF) approximation, and other mean-field methods have difficulties under these circumstances because of the presence of strong fluctuations. In principle, Coulomb interaction effects can be taken into account in a systematic way by using nonequilibrium Green function techniques, ${ }^{5,6}$ similar to the equilibrium case. Unfortunately, this technique becomes numerically very complicated in a strongly nonequilibrium situation, since in this case, the Green functions depend on two or more time arguments, which places high demands on the computer memory size and makes the numerical analysis very time consuming. ${ }^{1,7}$

In this paper, we discuss an alternative and potentially very powerful approach to study these kinds of problems, based on density-functional theory (DFT), ${ }^{8}$ and, in particular, its time-dependent generalization (TDDFT). ${ }^{9-11}$ In this approach, numerical calculations should be much less time consuming compared to the Green function method. TDDFT has been successfully applied to describe molecular excitations; ${ }^{12}$ however, this approach has some difficulties in describing extended systems. ${ }^{13}$ It is known that the standard local-density approximation (LDA) and generalized gradient approximation (GGA) for the DFT exchange-correlation (xc) potential cannot be applied to describe some effects beyond the ground-state properties in extended systems, such as the energy band gaps and excitonic effects in the optical absorption spectra. Therefore, in order to apply TDDFT to study ultrafast processes and, in particular, to describe correctly the optical absorption spectra in such systems, it is necessary to find suitable xc potentials.

For weak and smooth external fields, such a potential can be constructed by using the many-body Bethe-Salpeter equation (BSE) approach. ${ }^{14-16}$ In fact, in this case, TDDFT calculations with an xc potential extracted from the BSE give very good results for the optical absorption spectra in bulk semiconductors. Unfortunately, the BSE-TDDFT approach cannot be used directly to construct an xc potential for excitation with strong short pulses, since, here, the linear response theory cannot be applied, and the equations in time domain depend on many time variables, which makes numerical solution extremely difficult (similar to nonequilibrium Green's functions). Therefore, it would be extremely useful to find a simple xc potential which will allow one to 
use TDDFT straightforwardly to study the optical response of a system in the time domain. Kim and Görling ${ }^{17,18}$ have shown in frequency-dependent linear response that such an xc potential exists, namely, the time-dependent optimized effective potential in exact-exchange approximation (XXTDOEP), which gives rise to optical absorption spectra with pronounced excitonic features. We also mention that the XXTDOEP has recently been extended into the nonlinear, realtime domain for simple quasi-one-dimensional quantum-well systems. ${ }^{19}$

According to Kim and Görling, ${ }^{17,18}$ the main reason for the successful description of excitons in XX-TDOEP is the presence of a $1 / q^{2}$ Coulomb singularity in the xc kernel $f_{x c}\left(\mathbf{r}, t, \mathbf{r}^{\prime}, t^{\prime}\right)=\delta V_{x c}(\mathbf{r}, t) /\left.\delta n\left(\mathbf{r}^{\prime}, t^{\prime}\right)\right|_{n=n_{0}(\mathbf{r})}$ in momentum representation. However, in their calculations, it was crucial to introduce a cutoff of the long-range Coulomb interaction to prevent collapse of the spectra. This is essentially equivalent to using a screened Coulomb interaction, as discussed in detail by Bruneval et al. ${ }^{20}$

The purpose of this paper is twofold. (1) We will formulate a general TDDFT approach to study optical interband excitations in terms of the Kohn-Sham density matrix. The corresponding system of equations has the formal simplicity of the SBEs, which allows one to solve it directly in the time domain, contrary to the many-body Green function approach, where it is difficult to treat the problem numerically. At the same time, this approach has a great formal advantage in comparison with the SBE formalism, since in TDDFT, the Coulomb interaction effects are treated, in principle, exactly. (2) We will show that by using simple exchange-only functionals, the essence of excitonic features can be captured in a relatively simple manner. As an example, we consider the model of a three-dimensional two-band bulk insulator for different local xc potentials and show that its optical absorption spectra contain qualitatively correct excitonic features, whenever the xc energy kernel has a $1 / q^{2}$ singularity.

The paper is organized as follows. We introduce a general TDDFT formalism in terms of the Kohn-Sham density matrix in Sec. II. In Sec. III, we derive the TDDFT-SBE formalism and in Sec. IV, we apply this formalism to study the optical absorption spectra for a three-dimensional two-band model insulator with different xc potentials and compare the results with HF. Conclusions are presented in Sec. V. Some technical details are given in the Appendix. We use Hartree atomic units $\left(e^{2}=m=\hbar=1\right)$ throughout this paper.

\section{GENERAL FORMALISM}

The general DFT Hamiltonian for a many-electron system in a solid (in the Born-Oppenheimer approximation) can be written in the following form:

$$
\hat{H}=-\frac{\nabla^{2}}{2}+V_{\text {nucl }}(\mathbf{r})+V_{H}[n](\mathbf{r})+V_{x c}[n](\mathbf{r}),
$$

where $V_{\text {nucl }}(\mathbf{r})$ is the nuclear potential for the electrons and

$$
V_{H}[n](\mathbf{r})=\int d \mathbf{r}^{\prime} \frac{n\left(\mathbf{r}^{\prime}\right)}{\left|\mathbf{r}-\mathbf{r}^{\prime}\right|}
$$

is the Hartree potential, where $n(\mathbf{r})$ is the density of electrons. All many-body effects beyond Hartree are described by the scalar xc potential $V_{x c}[n](\mathbf{r})$. In particular, in the LDA exchange-only case:

$$
V_{x L D A}[n(\mathbf{r})]=-\left(\frac{3}{\pi}\right)^{1 / 3} n^{1 / 3}(\mathbf{r}) .
$$

In order to describe the ground-state properties of the system governed by the Hamiltonian in Eq. (1), one solves the stationary Kohn-Sham (KS) equation:

$$
\hat{H}(\mathbf{r}) \psi_{\mathbf{k}}^{l_{i}}(\mathbf{r})=\varepsilon_{\mathbf{k}}^{l_{i}} \psi_{\mathbf{k}}^{l_{i}}(\mathbf{r}),
$$

and find bands $l_{i}$ with spectra $\varepsilon_{\mathbf{k}}^{l_{i}}$, where $\mathbf{k}$ is the crystal momentum and $l_{i}=v_{i}, c_{i}(i=1,2, \ldots)$ are the labels for the valence $(v)$ and conduction $(c)$ bands. The electron density can be found self-consistently:

$$
n(\mathbf{r})=2 \sum_{i, \mathbf{k}}\left|\psi_{\mathbf{k}}^{v_{i}}(\mathbf{r})\right|^{2} \theta\left(\varepsilon_{F}-\varepsilon_{\mathbf{k}}^{v_{i}}\right),
$$

where $\varepsilon_{F}$ is the Fermi energy and the summation is performed over the occupied (valence) band states. Equation (5) is valid in the case of zero temperature, which we consider in this paper. Finite temperatures would require introduction of the Fermi distribution function into Eq. (5).

In order to study the nonequilibrium case when an external electric field $\mathbf{E}(t)$ is switched on at time $t=t_{0}$, the Hamiltonian in Eq. (1) must be modified in the standard way: an external electromagnetic vector potential $\mathbf{A}_{e x t}(\mathbf{r}, t)$ should be added by making the usual substitution $\nabla \rightarrow \nabla$ $-(i / c) \mathbf{A}_{e x t}(\mathbf{r}, t)$ and by adding a scalar potential term $\varphi_{e x t}(\mathbf{r}, t)$ to the Hamiltonian. The electric field is connected with $\varphi_{e x t}(\mathbf{r}, t)$ and $\mathbf{A}_{e x t}(\mathbf{r}, t)$ in the following way:

$$
\mathbf{E}(\mathbf{r}, t)=-\nabla \varphi_{e x t}(\mathbf{r}, t)-\frac{1}{c} \frac{\partial \mathbf{A}_{e x t}(\mathbf{r}, t)}{\partial t} .
$$

For an extended system, one needs to preserve the periodicity; therefore, an external vector potential must be used. This makes the problem technically more complicated in comparison to scalar potentials. However, it can be shown that in situations when the characteristic field frequency is bigger than the level spacing, one can work with scalar potentials instead of vector potentials (see, for example, Ref. 21). Therefore, we shall consider the case when $\mathbf{A}_{e x t}(\mathbf{r}, t)=0$ and, for simplicity, assume that the electric field is space independent. Strictly speaking, for external homogeneous fields, one needs to use current TDDFT in order to study the response of the system. 22,23 One then gets the macroscopic current, which allows one to satisfy the periodicity condition. In this paper, however, we use the usual approximation ${ }^{4}$ where the external homogeneous electric field is described by the following scalar potential:

$$
\varphi_{\text {ext }}(\mathbf{r}, t)=-\mathbf{E}(t) \mathbf{r} .
$$


Due to the presence of an electric field, the Hamiltonian is explicitly time dependent. Moreover, the Hartree and xc potential terms in the Hamiltonian also become time dependent. Such a time-dependent problem is described by the timedependent KS equation:

$$
i \frac{\partial}{\partial t} \Psi(\mathbf{r}, t)=\hat{H}(\mathbf{r}, t) \Psi(\mathbf{r}, t) .
$$

In the adiabatic approximation, which we use in this paper, the xc potentials can be formally obtained from the stationary potentials by simply evaluating them with the timedependent electron density, $n(\mathbf{r}) \rightarrow n(\mathbf{r}, t)$. Equation (8) must be solved self-consistently together with the corresponding time-dependent particle number equation [Eq. (5)]. Since the initially occupied states are the valence states $\psi_{\mathbf{k}}^{\nu_{i}}(\mathbf{r})$, described by the band index $v^{i}$ and the momentum $\mathbf{k}$, the evolution of the system can be completely described by the time evolution of these states, i.e., by finding the corresponding time-dependent wave functions $\Psi_{\mathbf{k}}^{v_{i}}(\mathbf{r}, t)$, such that $\Psi_{\mathbf{k}}^{v_{i}}\left(\mathbf{r}, t_{0}\right)=\psi_{\mathbf{k}}^{v_{i}}(\mathbf{r})$, from Eq. (8) and a time-dependent generalization of Eq. (5):

$$
n(\mathbf{r}, t)=2 \sum_{i, \mathbf{k}}\left|\Psi_{\mathbf{k}}^{v_{i}}(\mathbf{r}, t)\right|^{2} \theta\left(\varepsilon_{F}-\varepsilon_{\mathbf{k}}^{v_{i}}\right) .
$$

In the following, we express the time-dependent wave functions as linear combinations of the ground-state wave functions:

$$
\Psi_{\mathbf{k}}^{v_{i}}(\mathbf{r}, t)=\sum_{j, \mathbf{q}}\left[c_{\mathbf{k} \mathbf{q}}^{v_{i} v_{j}}(t) \psi_{\mathbf{q}}^{v_{j}}(\mathbf{r})+c_{\mathbf{k q}}^{v_{i} c_{j}}(t) \psi_{\mathbf{q}}^{c_{j}}(\mathbf{r})\right]
$$

where $c_{\mathbf{k} \mathbf{q}_{i}}^{v_{i} l}(t)$ are momentum and time-dependent complex coefficients, which satisfy the following initial condition: $c_{\mathbf{k} \mathbf{q}}^{v_{i} l_{j}}\left(t_{0}\right)=\delta_{\mathbf{k q}} \delta_{v_{i} l j} e^{-i \varepsilon_{\mathbf{k}}^{v_{i}} t_{0}}$. The time evolution of the system can thus be found by determining the coefficients $c_{\mathbf{k q}}^{v_{i} v_{j}}(t)$ and $c_{\mathbf{k q}}^{v_{i} c_{j}}(t)$ for Eq. (10). However, in order to solve the problem, it is more convenient to introduce the density matrix: $\rho_{\mathbf{k} ; \mathbf{q} p}^{v_{i} ;} l_{m} \bar{l}_{n}(t)=c_{\mathbf{k q}}^{v_{i} l_{m}}(t)\left[c_{\mathbf{k} p}^{v_{i} \bar{l}_{n}}(t)\right]^{*}$, which is useful for defining physical quantities such as occupation of the states and optical transitions (see next section). TDDFT in the densitymatrix representation is a method which allows one to solve the problem, in principle, exactly, since it is an exact reformulation of the time-dependent Kohn-Sham formalism. This method was already introduced to study intersubband processes in quantum wells ${ }^{24}$ and the electrical conductivity in dissipative models of molecular devices ${ }^{25}$ (see also Ref. 26, where a similar approach was discussed). Here, we develop a technique which can be applied in more general cases with a continuous electron spectrum, including interband transitions in solids.

The density matrix satisfies the following equation of motion:

$$
\begin{aligned}
& i \frac{\partial}{\partial t} \rho_{\mathbf{k} ; \mathbf{q} p}^{v_{i} ; l_{n} l_{n}^{\prime}}(t)=[H(t), \rho]_{\mathbf{k} ; \mathbf{q} \mathbf{p}}^{v_{i} ; l_{m} l_{n}^{\prime}}
\end{aligned}
$$

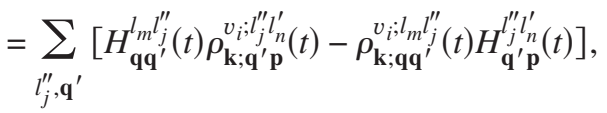

where the Hamiltonian matrix elements $H_{\mathbf{k q}}^{l_{m} \bar{l}_{n}}(t)$ are

$$
\begin{aligned}
H_{\mathbf{k q}}^{l_{m} l_{n}^{\prime}}(t) & =\int_{\text {cell }} d \mathbf{r} \psi_{\mathbf{k}}^{l_{m}^{*}}(\mathbf{r}) H(t) \psi_{\mathbf{q}}^{l_{n}^{\prime}}(\mathbf{r}) \\
& =\varepsilon_{\mathbf{k}}^{l_{m}} \delta_{l_{m} l_{n}^{\prime}} \delta_{\mathbf{k q}}+\mathbf{E}(t) \mathbf{d}_{\mathbf{k q}}^{l_{m} l_{n}^{\prime}}+V_{H \mathbf{k q}}^{l_{m} l_{n}^{\prime}}(t)+V_{x \mathbf{k q}}^{l_{m} l_{n}^{\prime}}(t),
\end{aligned}
$$

and the space integration is performed over a unit cell. In Eq. (12),

$$
\mathbf{d}_{\mathbf{k q}}^{l_{m} l_{n}^{\prime}}=\int_{\text {cell }} d \mathbf{r} \psi_{\mathbf{k}}^{l_{m}^{*}}{ }^{*}(\mathbf{r}) \mathbf{r} \psi_{\mathbf{q}}^{l_{n}^{\prime}}(\mathbf{r})
$$

are the dipole matrix elements, ${ }^{21}$ and $V_{H \mathbf{k q}}^{l_{m} l_{n}^{\prime}}(t)$ and $V_{x c \mathbf{k q}} l_{m} l_{n}^{\prime}(t)$ are the matrix elements for the difference between the timedependent and the ground-state (at $t \leqslant t_{0}$ ) Hartree and xc potentials:

$$
V_{H \mathbf{k q}}^{l_{l} l_{n}^{\prime}}(t)=\int_{\text {cell }} d \mathbf{r} \psi_{\mathbf{k}}^{l_{\mathbf{k}}{ }^{*}}(\mathbf{r})\left\{V_{H}[n](\mathbf{r}, t)-V_{H}[n]\left(\mathbf{r}, t_{0}\right)\right\} \psi_{\mathbf{q}}^{l^{\prime}}(\mathbf{r}),
$$

and similar for $V_{x c \mathbf{k q}}^{l_{m} l_{n}^{\prime}}(t)$. The density matrix satisfies the following initial condition:

$$
\rho_{\mathbf{k} ; \mathbf{q}}^{v_{i} ; l_{m} l_{n}^{\prime}}\left(t_{0}\right)=\delta_{\mathbf{k q}} \delta_{\mathbf{k p}} \delta_{v_{i} l_{m}} \delta_{v_{i} l_{n}^{\prime}},
$$

which corresponds to the situation where all states in the valence bands are initially occupied. The particle density in Eq. (9) has the following form in terms of the density-matrix elements:

$$
n(\mathbf{r}, t)=2 \sum_{i, l_{m}, l_{n}^{\prime}, \mathbf{k}, \mathbf{q}, \mathbf{p}} \rho_{\mathbf{k}, \mathbf{q}, \mathbf{p}}^{v_{i} l_{m} l_{n}^{\prime}}(t) \psi_{\mathbf{p}}^{l^{\prime} * *}(\mathbf{r}) \psi_{\mathbf{q}}^{l_{m}}(\mathbf{r}) \theta\left(\varepsilon_{F}-\varepsilon_{\mathbf{k}}^{v_{i}}\right)
$$

The solution of the Liouville-von Neumann equation [Eq. (11)] allows one to study the physical properties of the system. In particular, one can find the dynamic polarization as

$$
\mathbf{D}(t)=\sum_{i, l_{m}, l_{n}^{\prime}, \mathbf{k}, \mathbf{q}, \mathbf{p}} \rho_{\mathbf{k} ; \mathbf{q}}^{v_{i}, l_{m} l_{n}^{\prime}}(t) \mathbf{d}_{\mathbf{p q}}^{l_{n}^{\prime} l_{m}}
$$

Below, we shall use this formalism to study the optical response of a three-dimensional two-band insulator by solving Eqs. (11) and (15) using different xc potentials.

\section{THREE-DIMENSIONAL TWO-BAND MODEL}

In general, it is very difficult to find the solution of the density-matrix equation [Eq. (11)] and one needs to make some approximations. For simplicity, we shall consider optical absorption spectra of systems composed of one valence 
band and one conduction band: $l_{1}=v, l_{2}=c$, i.e., we assume that the dominant optical transitions in the system take place from the highest occupied valence band to the lowest conduction band. This widely used approximation is quite accurate as long as the electric field is not too strong so that multiphoton processes come into play and as long as the laser pulse bandwidth remains much smaller than the band gap, which is usually the case. In the present context, since the main aim of this paper is a proof of principle that TDDFT can describe excitonic effects in semiconductors triggered by short laser pulses, a two-band model is sufficient. However, for real systems, one may need to take into account the band structure of the materials more accurately. Another simplification comes from the fact that in the dipole approximation for the external field, the optical transitions in the system take place with zero photon momentum. The coefficients $c$ [defined in Eq. (10)] then depend on one momentum variable only:

$$
c_{\mathbf{k q}}^{v v}(t)=\delta_{\mathbf{k q}} c_{\mathbf{k}}^{v v}(t), \quad c_{\mathbf{k q}}^{v c}(t)=\delta_{\mathbf{k} \mathbf{q}} c_{\mathbf{k}}^{v c}(t) .
$$

The problem is thus reduced to finding the density matrix of rank 2, with elements $\rho_{\mathbf{k}}^{v v}(t), \rho_{\mathbf{k}}^{v c}(t), \rho_{\mathbf{k}}^{c v}(t)$, and $\rho_{\mathbf{k}}^{c c}(t)$, which are functions of momentum and time. The elements $\rho_{\mathbf{k}}^{v v}(t)$ and $\rho_{\mathbf{k}}^{c c}(t)$ describe the occupancy of the valence and conduction band states, and $\rho_{\mathbf{k}}^{v c}(t)$ and $\rho_{\mathbf{k}}^{c v}(t)$ describe the polarization in the system. These four elements are not independent: particle number conservation requires $\rho_{\mathbf{k}}^{v v}(t)+\rho_{\mathbf{k}}^{c c}(t)=1$, and by definition, $\rho_{\mathbf{k}}^{c v}(t)=\rho_{\mathbf{k}}^{v c^{*}}(t)$.

From Eq. (11), one then obtains the following system of equations for two independent components $\rho_{\mathbf{k}}^{v v}(t)$ and $\rho_{\mathbf{k}}^{v c}(t)$ :

$$
\begin{aligned}
\frac{\partial}{\partial t} \rho_{\mathbf{k}}^{v v}(t) & =-2 \operatorname{Im}\left\{\left[E(t) d_{\mathbf{k}}^{c v}+V_{H \mathbf{k}}^{c v}+V_{x c \mathbf{k}}^{c v}\right] \rho_{\mathbf{k}}^{v c}(t)\right\}, \\
\frac{\partial}{\partial t} \rho_{\mathbf{k}}^{v c}(t)= & -i\left[\varepsilon_{\mathbf{k}}^{v}-\varepsilon_{\mathbf{k}}^{c}\right] \rho_{\mathbf{k}}^{v c}(t)-i\left[\rho_{\mathbf{k}}^{c c}(t)-\rho_{\mathbf{k}}^{v v}(t)\right] E(t) d_{\mathbf{k}}^{v c} \\
& -i\left[\rho_{\mathbf{k}}^{c c}(t)-\rho_{\mathbf{k}}^{v v}(t)\right]\left[V_{H \mathbf{k}}^{v c}(t)+V_{x c \mathbf{k}}^{v c}(t)\right] \\
& -i\left[V_{H \mathbf{k}}^{v v}(t)+V_{x c \mathbf{k}}^{v v}(t)-V_{H \mathbf{k}}^{c c}(t)-V_{x c \mathbf{k}}^{c c}(t)\right] \rho_{\mathbf{k}}^{v c}(t) .
\end{aligned}
$$

This system represents the TDDFT version of the wellknown SBEs, ${ }^{4}$ which are used to study the optical properties of bulk semiconductors subject to external electric fields. Equations (19) and (20) have a more general form, since, here, the Coulomb interaction effects are taken into account, in principle, exactly by means of the matrix elements of $V_{x c}[n](\mathbf{r}, t)$. As mentioned in the Introduction, this is important in the case of small systems and sharp pulses, where the characteristic times in the system are shorter than the Coulomb scattering time and where it is difficult to treat the Coulomb interaction effects properly within the SBE approach.

We shall study the solution of Eqs. (19) and (20) for the simple but instructive example of a two-band model on a cubic lattice. We assume that the solution of Eq. (4) with the Hamiltonian in Eq. (1) gives the following simple dispersions:

$$
\begin{aligned}
& \varepsilon_{\mathbf{k}}^{v}=\varepsilon_{0}^{v}+2 t^{v}\left[\cos \left(a_{0} k_{x}\right)+\cos \left(a_{0} k_{y}\right)+\cos \left(a_{0} k_{z}\right)\right], \\
& \varepsilon_{\mathbf{k}}^{c}=\varepsilon_{0}^{c}-2 t^{c}\left[\cos \left(a_{0} k_{x}\right)+\cos \left(a_{0} k_{y}\right)+\cos \left(a_{0} k_{z}\right)\right],
\end{aligned}
$$

where $a_{0}$ is the lattice constant. For definiteness, we consider a model of solid hydrogen and assume that the difference of $\varepsilon_{0}^{c}$ and $\varepsilon_{0}^{v}$ can be set proportional to the first two hydrogen energy levels $E=-1 /\left[2 a_{B} n^{2}\right], n=1,2$. In the last equation, $a_{B}=\hbar^{2} / m e^{2}=1$ is the Bohr radius, which is used as the length unit in this paper. It is assumed that the band parameters $t_{v}$ and $t_{c}$ are much smaller than $\varepsilon_{0}^{c}-\varepsilon_{0}^{v}$, i.e., the bands are nonoverlapping.

The valence and conduction band wave functions in the Bloch representation:

$$
\begin{aligned}
& \psi_{\mathbf{k}}^{v}(\mathbf{r})=e^{i \mathbf{k r}} u_{\mathbf{k}}^{v}(\mathbf{r}), \\
& \psi_{\mathbf{k}}^{c}(\mathbf{r})=e^{i \mathbf{k r}} u_{\mathbf{k}}^{c}(\mathbf{r}),
\end{aligned}
$$

can be written as linear combinations of Wannier functions $w^{v}(\mathbf{r})$ and $w^{c}(\mathbf{r})$. In fact, one can use the following representation for spatially periodic Bloch functions $u_{\mathbf{k}}^{v}(\mathbf{r})$ and $u_{\mathbf{k}}^{v}(\mathbf{r})$ :

$$
u_{\mathbf{k}}^{v}(\mathbf{r})=\sum_{\mathbf{L}} e^{i \mathbf{k}(\mathbf{r}-\mathbf{L})} w^{v}(\mathbf{r}-\mathbf{L}),
$$

and similar for $u_{\mathbf{k}}^{c}(\mathbf{r})$, where $\mathbf{L}=a_{0}\left(n_{x}, n_{y}, n_{z}\right)$ are the unit cell vectors.

Unfortunately, it is difficult to find the exact expressions for the Wannier functions, and hence for the Bloch functions, even for such a simple three-dimensional system. For simplicity, we choose the Wannier functions to be equal to the $1 s^{0}$ and $2 p^{0}$ hydrogen wave functions:

$$
\begin{gathered}
w^{v}(\mathbf{r})=\frac{1}{\sqrt{\pi}} e^{-r}, \\
w^{c}(\mathbf{r})=\frac{1}{4 \sqrt{2 \pi}} e^{-r / 2} z .
\end{gathered}
$$

This choice of the Wannier functions is an approximation, since the orthogonality condition on different sites $\int d \mathbf{r} w^{l^{*}}\left(\mathbf{r}-\mathbf{L}_{1}\right) w^{m}\left(\mathbf{r}-\mathbf{L}_{2}\right)=\delta^{l m} \delta_{\mathbf{L}_{1} \mathbf{L}_{2}}$ is violated. However, when the lattice parameter $a_{0}$ is much larger than the Bohr radius $a_{B}$, the requirement of orthogonality is satisfied with a very high precision, since the overlap between different sites becomes negligible. In our calculations, we shall use the lattice constant values $a_{0}=10$ and 20 .

In the next section, we shall study the solution of Eqs. (19) and (20) when an external short-pulse field:

$$
\mathbf{E}(t)=\mathbf{E}_{0} e^{-t^{2} / \tau^{2}}
$$

with $\tau \sim 10-100 \mathrm{fs}$, is applied. We numerically propagate the time evolution of the system starting at a large negative time $t_{0}\left(\left|t_{0}\right| \gg \tau\right)$, which means that the pulse is a smooth continuous function of time. The optical absorption spectrum $A(\omega)$ is then obtained as the real part of the ratio of the Fourier transforms of the total polarization: 


$$
P(\omega)=i\left(4 \pi / \sqrt{\epsilon_{b}}\right)\left(\varepsilon_{0}^{c}-\varepsilon_{0}^{v}\right)\left|d^{c v}\right|^{2} a_{0}^{3} \int \frac{d \mathbf{k}}{(2 \pi)^{3}} \int d t e^{i \omega t} \rho_{\mathbf{k}}^{v c}(t),
$$

and the pulse field $E(\omega)$ :

$$
A(\omega)=-2 \operatorname{Re}[P(\omega) / E(\omega)] .
$$

\section{RESULTS AND DISCUSSION}

\section{A. Hartree-Fock}

Before proceeding with the solution of the TDDFT equations [Eqs. (19) and (20)] for different xc potentials, we discuss the case of the Hartree-Fock potential for the two-band model presented above. Hartree-Fock is the most widely used approximation in the SBE theory ${ }^{4}$ for the study of optical absorption spectra in bulk systems and heterostructures subject to both smooth and pulsed external fields (see, for example, Refs. 27 and 28).

In the time-dependent Hartree-Fock approximation, the total electron wave function satisfies the following equation:

$$
\begin{aligned}
i \frac{\partial \Psi_{\mathbf{k}}^{v}(\mathbf{r}, t)}{\partial t}= & {\left[-\frac{\nabla^{2}}{2}-\mathbf{E}(t) \mathbf{r}\right.} \\
& \left.+\int d \mathbf{r}^{\prime} \frac{\sum \Psi_{\mathbf{q}}^{v^{*}}\left(\mathbf{r}^{\prime}, t\right) \Psi_{\mathbf{q}}^{v}\left(\mathbf{r}^{\prime}, t\right)}{\left|\mathbf{r}-\mathbf{r}^{\prime}\right|}\right] \Psi_{\mathbf{k}}^{v}(\mathbf{r}, t) \\
& -\int d \mathbf{r}^{\prime} \frac{\sum \Psi_{\mathbf{q}}^{v^{*}}\left(\mathbf{r}^{\prime}, t\right) \Psi_{\mathbf{q}}^{v}(\mathbf{r}, t)}{\left|\mathbf{r}-\mathbf{r}^{\prime}\right|} \Psi_{\mathbf{k}}^{v}\left(\mathbf{r}^{\prime}, t\right) .
\end{aligned}
$$

Applying the analysis presented in Sec. II [see Eqs. (10)-(16)], it is possible to show that Eq. (31) is equivalent to the system of TDDFT density-matrix equations [Eqs. (19) and (20)] with corresponding matrix elements for the Hartree and Hartree-Fock potentials. Namely, the Hartree potential matrix elements are

$$
V_{H \mathbf{k}}^{l m}(t)=\sum_{n, s=v, c ; \mathbf{q}} I_{H}^{l m n s}(\mathbf{k}, \mathbf{q}) \rho_{\mathbf{q}}^{s n}(t),
$$

where

$$
I_{H}^{l m n s}(\mathbf{k}, \mathbf{q})=\int d \mathbf{r} \int d \mathbf{r}^{\prime} \psi_{\mathbf{k}}^{l^{*}}(\mathbf{r}) \psi_{\mathbf{k}}^{m}(\mathbf{r}) \frac{\psi_{\mathbf{q}}^{n^{*}}\left(\mathbf{r}^{\prime}\right) \psi_{\mathbf{q}}^{s}\left(\mathbf{r}^{\prime}\right)}{\left|\mathbf{r}-\mathbf{r}^{\prime}\right|} .
$$

From Eqs. (32) and (33), one can find the following approximate expression for the Hartree matrix elements:

$V_{H \mathbf{k}}^{l m}(t)=\delta^{l m} V(\mathbf{G} \rightarrow \mathbf{0})+\sum_{n, s=v, c ; \mathbf{q}} A^{l m n s} a_{0}^{3} \int \frac{d \mathbf{q}}{(2 \pi)^{3}} \rho_{\mathbf{q}}^{s n}(t)+\cdots$,

where $V(\mathbf{q})$ is the Fourier transform of the Coulomb potential $1 /\left|\mathbf{r}-\mathbf{r}^{\prime}\right|, \mathbf{G}$ is a reciprocal lattice vector, and

$$
\begin{aligned}
A^{l m n s}= & \sum_{\mathbf{G} \neq 0} V(\mathbf{G}) \int d \mathbf{r} w^{l l^{*}}(\mathbf{r}) e^{-i \mathbf{G r}} w^{m}(\mathbf{r}) \\
& \times \int d \mathbf{r}^{\prime} w^{n}\left(\mathbf{r}^{\prime}\right) e^{i \mathbf{G r} \mathbf{r}^{\prime}} w^{s}\left(\mathbf{r}^{\prime}\right)
\end{aligned}
$$

(details of a similar analysis can be found in Ref. 21). In Eq. (34), the dots correspond to the terms proportional to integrals over products of Wannier functions which reside on different sites $\left[\sim \int d \mathbf{r} w^{l^{*}}(\mathbf{r}) w^{m}(\mathbf{r}-\mathbf{L})\right]$. These terms are negligible due to a small overlap of the Wannier functions on different sites [when $a_{B} \ll a_{0}$, see Eqs. (26) and (27)]. Moreover, the presence of oscillating functions $\exp [-i \mathbf{G r}]$ and $\exp \left[i \mathbf{G} \mathbf{r}^{\prime}\right]$ under the integrals in expression (35) for the coefficients $A^{l m n s}$ makes the terms proportional to $A^{l m n s}$ much smaller than the first term in Eq. (34). Therefore, the second term in Eq. (34) can also be neglected. Substitution of the remaining term $\delta^{l m} V(\mathbf{G} \rightarrow \mathbf{0})$ into Eqs. (19) and (20) simply causes a constant shift of the overall potential and can be ignored. Therefore, the Hartree term does not contribute to the density-matrix equations [Eqs. (19) and (20)] in this approximation, and we shall ignore it from now on. It is possible to show ${ }^{21}$ that higher order terms in Eq. (34), which we neglect, lead to a small renormalization of the energy bands and the electric field time dependence.

In a similar way, one can find an approximate expression for the matrix elements of the nonlocal Fock exchange potential:

$$
V_{H F \mathbf{k}}^{l m}(t)=-a_{0}^{3} \int \frac{d \mathbf{q}}{(2 \pi)^{3}} V(\mathbf{k}-\mathbf{q}) \rho_{\mathbf{q}}^{l m}(t) .
$$

Substitution of expression (36) into Eqs. (19) and (20) leads to the following density-matrix equations in the HartreeFock approximation:

$$
\begin{aligned}
\frac{\partial}{\partial t} \rho_{\mathbf{k}}^{v v}(t)= & -2 \operatorname{Im}\left[\left(E(t) d_{\mathbf{k}}^{c v}+a_{0}^{3} \int \frac{d \mathbf{q}}{(2 \pi)^{3}} V(\mathbf{k}\right.\right. \\
& \left.\left.-\mathbf{q}) \rho_{\mathbf{q}}^{c v}(t)\right) \rho_{\mathbf{k}}^{v c}(t)\right], \\
\frac{\partial}{\partial t} \rho_{\mathbf{k}}^{v c}(t)= & -i\left\{\varepsilon_{\mathbf{k}}^{v}-\varepsilon_{\mathbf{k}}^{c}-a_{0}^{3} \int \frac{d \mathbf{q}}{(2 \pi)^{3}} V(\mathbf{k}-\mathbf{q})\left[\rho_{\mathbf{q}}^{v v}(t)\right.\right. \\
- & \left.\left.\rho_{\mathbf{q}}^{c c}(t)\right]\right\} \rho_{\mathbf{k}}^{v c}(t)-i\left[\rho_{\mathbf{k}}^{c c}(t)-\rho_{\mathbf{k}}^{v v}(t)\right]\left(E(t) d_{\mathbf{k}}^{v c}\right. \\
& \left.-a_{0}^{3} \int \frac{d \mathbf{q}}{(2 \pi)^{3}} V(\mathbf{k}-\mathbf{q}) \rho_{\mathbf{q}}^{v c}(t)\right) .
\end{aligned}
$$

These equations are equivalent to the standard SBEs. ${ }^{4}$ The solution of the system of Eqs. (37) and (38) in the presence of an external field $\mathbf{E}(t)$ allows one to calculate the optical polarization in the system by means of Eq. (30).

In Fig. 1, we present the frequency dependence of the optical absorption spectra in Hartree-Fock approximation when an external short-time pulse of the form of Eq. (28) is applied. The calculations are for different values of the valence and conduction bandwidths $W^{v}$ and $W^{c}$. We find pro- 


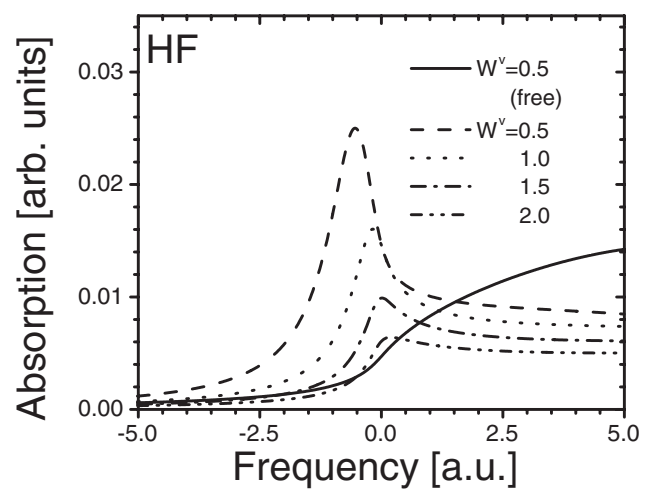

FIG. 1. Hartree-Fock optical absorption spectra for parabolic bands at different values of the valence bandwidth $W^{v}$ (divided by $\pi^{2}$ ). We consider external pulses of the form of Eq. (28), with pulse duration $\tau=0.05$ and electric field amplitude $E_{0}=2.0$. The conduction bandwidth and the band gap are $W^{c}=0.4 \pi^{2}$ and $\omega_{g}=5$. Frequencies are measured with respect to $\omega_{g}$.

nounced excitonic peaks whose strength increases with decreasing valence bandwidth.

Here and in other figures, we introduce a screening parameter $\lambda=1$ in the Coulomb kernel $1 /\left(\mathbf{q}^{2}+\lambda^{2}\right)$. As we already discussed in the Introduction, screening is an important feature in exchange-only calculations, since it prevents the excitonic absorption spectrum from collapsing. Introducing a screening (or cutoff) parameter significantly improves the agreement with experiment. ${ }^{17,20}$

Furthermore, in order to reach a steady state more quickly, we introduce decoherence terms $\Gamma(1+\alpha|\mathbf{k}| / \pi) \rho^{l m}$ on the right-hand side of the density-matrix equations (we choose $\Gamma=0.2$ and $\alpha=7.5)$. In this paper, in the numerical results for the absorption spectra, we set the prefactor $\left(8 \pi / \sqrt{\epsilon_{b}}\right)\left(\varepsilon_{0}^{c}\right.$ $\left.-\varepsilon_{0}^{v}\right)\left|d^{c v}\right|^{2}$ to be equal to 1 [see Eqs. (29) and (30)]. The absorption spectra demonstrate pronounced excitonic features with shape and peak positions that depend on the external field amplitude parameters.

The SBE approach was successfully applied to study optical properties of different bulk materials in the case of weak external fields, when linear response theory can be applied. Recently, this formalism was used to study the nonlinear response of various quantum-well systems ${ }^{27,28}$ and some other effects, such as the sideband generation, Franz-Keldysh effect, four-wave mixing, and higher correlation effects, in particular, biexcitons. ${ }^{3}$

As discussed in the Introduction, the main shortcoming of the SBE approach for small (nano)systems and short pulses is that the Coulomb interaction effects are treated on a meanfield theory level. All fluctuation effects are hidden in the inverse scattering (decoherence) time parameters $\Gamma_{\mathbf{k}}^{l m}$, which are usually introduced into the system of the SBEs [Eqs. (37) and (38)] by means of the terms $\Gamma_{\mathbf{k}}^{l m} \rho_{\mathbf{k}}^{l m}$. However, the characteristic times in this case are shorter than the Coulomb scattering time; therefore, fluctuation effects are very important and cannot be neglected. It is extremely difficult to include higher Coulomb terms in the SBEs in a controllable way, since in this case, one needs to consider additional equations for higher order correlation functions. The TDDFT

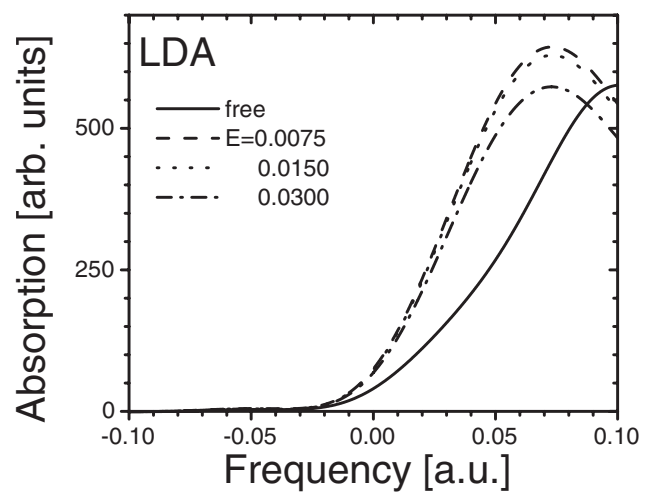

FIG. 2. Absorption spectra in ALDA at different values of the electric field amplitude and the free electron bands $W^{v}=0.15$, $W^{c}=0.06$. The other parameters are $\omega_{g}=0.375, a_{0}=20, \tau=10$, $\Gamma=0.0025$, and $\alpha=7.5$.

approach allows one to treat the Coulomb effects, in principle, exactly, which should make this approach favorable in the case of small systems and short-time pulses, provided one can find a suitable xc functional to account for correlations. In the following, we shall consider the optical properties of our two-band insulator by means of the TDDFT formalism developed in Sec. II. We limit ourselves here to exchange-only functionals.

\section{B. Adiabatic local density approximation and generalized gradient approximation}

The LDA is a standard approximation used in DFT to study the ground-state properties of many materials. In the time-dependent case, there exists a generalization of this approximation - the adiabatic LDA (ALDA). In the adiabatic approximation, it is assumed that the xc potential at time $t$ depends on the time-dependent particle density $n(\mathbf{r}, t)$ at the same time $t$ only. In other words, all memory effects are neglected, and the TDDFT equations remain local in time, which allows one to solve the problem numerically relatively easily. In ALDA, the x-only potential matrix elements have the following momentum and time dependence:

$$
V_{x L D A \mathbf{k}}^{l m}(t)=-\left(\frac{3}{\pi}\right)^{1 / 3} \int_{\text {cell }} d \mathbf{r} \psi_{\mathbf{k}}^{l^{*}}(\mathbf{r}) n^{1 / 3}(\mathbf{r}, t) \psi_{\mathbf{k}}^{m}(\mathbf{r})
$$

Our numerical analysis confirms the previous observation $^{1}$ that the optical absorption spectra do not demonstrate excitonic features with the ALDA exchange potential in Eq. (39) at various values of the model parameters (Fig. 2). Actually, there is a very wide peak in the absorption spectra at rather large values of the quasiparticle masses (or very narrow energy bands); however, this wide absorption spectral weight cannot be related to a well-defined excitonic energy value and, hence, it does not correspond to an exciton formation. For details of the numerical calculations in ALDA and other potentials used in this paper (see below), we refer the reader to the Appendix.

Similar results can be obtained for standard adiabatic GGAs, for example, the van Leeuwen-Baerends ${ }^{29}$ (LB) po- 
tential. In the LB approximation, the xc potential has the following form:

$$
V_{L B}(\mathbf{r}, t)=V_{L D A}(\mathbf{r}, t)-\beta n^{1 / 3}(\mathbf{r}, t) \frac{x^{2}}{1+3 \beta x \ln \left(x+\sqrt{x^{2}+1}\right)},
$$

where $x=|\nabla n(\mathbf{r}, t)| / n^{4 / 3}(\mathbf{r}, t)$ and $\beta$ is a material-dependent parameter. We choose this particular GGA, since it has an important feature for finite systems, namely, a correct $(\sim 1 / r)$ asymptotic behavior at long distances (the ALDA xc potential and most standard GGAs decrease exponentially at $r \rightarrow \infty)$. The LB has been widely used in calculations of highly excited states, dipole moments, and other densitydependent properties. However, these desirable features do not seem to carry over for extended systems: no excitonic features are produced in our calculations.

Thus, we have seen that the standard adiabatic LDA + GGA for the xc potentials does not allow one to reproduce the excitonic features in the optical absorption spectra within the TDDFT formalism developed above. One needs to go beyond this approximation and find another class of simple TDDFT potentials which demonstrate excitonic peaks in the absorption spectra. As shown by Kim and Görling, ${ }^{17,18}$ such potentials exist. The main requirement is a $1 / q^{2}$ singularity in the exchange energy kernel. In the following, we shall consider the optical absorption spectra for classes of functionals where the underlying exchange energy has such a singularity.

\section{Slater and Krieger-Li-Iafrate potentials}

As shown above, the ALDA cannot describe excitonic effects in the optical absorption spectra. A fundamental shortcoming of the LDA is that it contains a self-interaction error. In order to reduce this error, several approximations for self-interaction corrected (SIC) potentials were proposed. Probably the most often used SIC potential is due to Perdew and Zunger. ${ }^{30}$ However, in their scheme, one must deal with orbital-dependent potentials, which makes the calculations difficult, especially in the time-dependent case. To make a computational procedure simpler, the method of the optimized effective potential (OEP) was proposed (for overview and references, see, for example, Ref. 31). In this approach, all the wave functions for different orbitals satisfy a Schrödinger equation with a common, orbital-independent xc potential $v_{x c \sigma}^{O E P}(\mathbf{r})$. A time-dependent generalization of the OEP method (TDOEP) was given in Refs. 10 and 32. Unfortunately, the full TDOEP is computationally very demanding and has only recently been solved for simple quasi-onedimensional quantum-well systems. ${ }^{19}$

Krieger, et $a l^{33}$ proposed a simplified method [the Krieger-Li-Iafrate (KLI) scheme] for the OEP in the equilibrium exact-exchange case with $v_{x c \sigma}^{O E P}(\mathbf{r})$ depending explicitly on the orbital functions $\varphi_{j \sigma}$. In the equilibrium case, the KLI potential is defined by the following integral equation:

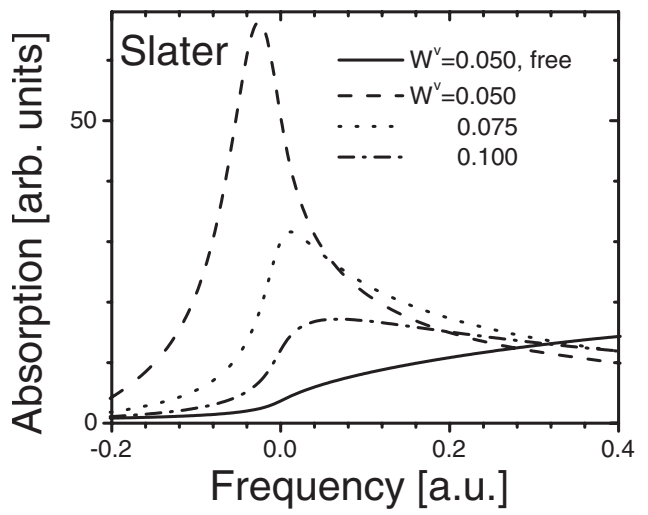

FIG. 3. Optical absorption spectra in the Slater approximation at different values of the valence bandwidth (divided by $\pi^{2}$ ). The other parameters are $W^{c}=0.025 \pi^{2}, \omega_{g}=0.2, a_{0}=10, \tau=4$, $E=0.0166, \Gamma=0.012$, and $\alpha=7.5$.

$$
\begin{aligned}
v_{x c \sigma}^{K L I}(\mathbf{r})= & \sum_{j} \frac{n_{j \sigma}(\mathbf{r})}{n_{\sigma}(\mathbf{r})}\left\{u_{x c j \sigma}(\mathbf{r})+\int d^{3} r^{\prime}\left|\varphi_{j \sigma}\left(\mathbf{r}^{\prime}\right)\right|^{2}\left[v_{x c \sigma}^{K L I}\left(\mathbf{r}^{\prime}\right)\right.\right. \\
& \left.\left.-u_{x c j \sigma}\left(\mathbf{r}^{\prime}\right)\right]\right\},
\end{aligned}
$$

where the orbital-dependent potentials $u_{x c j \sigma}(\mathbf{r})$ are obtained from the xc energy $E_{x c}$ as follows:

$$
u_{x c j \sigma}(\mathbf{r})=\left[f_{j \sigma} \varphi_{j \sigma}^{*}(\mathbf{r})\right]^{-1} \delta E_{x c}\left[\left\{\varphi_{j \sigma}\right\}\right] / \delta \varphi_{j \sigma}(\mathbf{r}) .
$$

This approximation was generalized to the time-dependent case in the adiabatic approximation $\left[\varphi_{j \sigma}(\mathbf{r}) \rightarrow \varphi_{j \sigma}(\mathbf{r}, t)\right]$ in Ref. 32.

In the linear regime, the optical absorption spectra of insulators show excitonic peaks within linearized TDOEP when an exchange energy kernel containing a $1 / q^{2}$ singularity is used. ${ }^{17,18}$ However, it is much more difficult to use this approach for short and strong pulses, when one needs to go beyond linear response.

Here, we consider simplified adiabatic TDOEPs with a Coulomb singularity in the exchange energy kernel, which are approximate solutions of Eqs. (41) and (42), and solve the problem in the time domain by using the density-matrix formalism developed in the previous sections. This approach can be applied for external fields of arbitrary strength and duration. However, as mentioned at the beginning of Sec. III, the two-band approximation used in this paper is valid when the field is not too strong.

We consider the time-dependent KLI and Slater potentials, ${ }^{33}$ where the latter is obtained as an approximation to KLI if one neglects the orbital-dependent constants on the right-hand side of Eq. (41):

$$
v_{x c \sigma}^{\text {Slater }}(\mathbf{r}, t)=\sum_{j} \frac{n_{j \sigma}(\mathbf{r}, t)}{n_{\sigma}(\mathbf{r}, t)} u_{x c j \sigma}(\mathbf{r}, t) .
$$

We use the Fock exchange energy for our two-band model in Eq. (42). Optical absorption spectra obtained with the Slater and KLI exchange potentials at different values of the model parameters are presented in Figs. 3 and 4. 


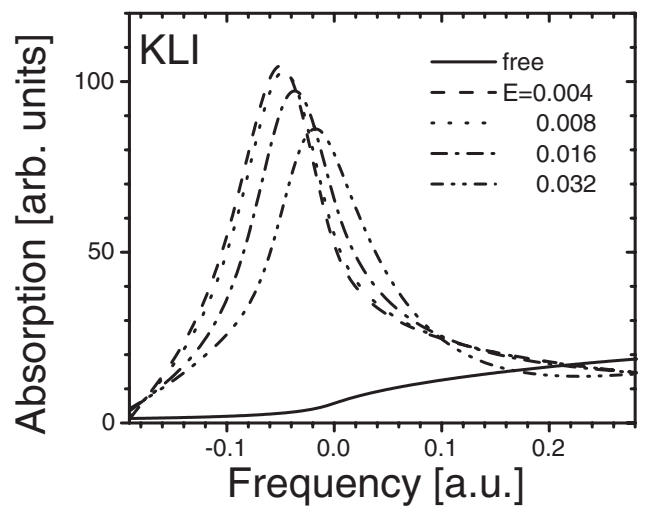

FIG. 4. Optical absorption spectra in the KLI approximation at different values of the electric field. The other parameters are $W^{v}$ $=0.0416 \pi^{2}, W^{c}=0.0208 \pi^{2}, \omega_{g}=0.166, a_{0}=10, \tau=4.8, \Gamma=0.012$, and $\alpha=7.5$.

As can be seen from these figures, the absorption spectra demonstrate a significant excitonic peak. In particular, the excitonic peak amplitude and the exciton binding energy decrease with increasing valence bandwidth in the Slater case (Fig. 3). This effect takes place because the increase of the bandwidth is equivalent to a reduction of the effective mass of electron-hole pairs. A similar behavior has been found in HF (see Fig. 1), and we have also observed it for KLI. Figure 4 shows the KLI absorption spectrum at different values of the electric field. Here, the spectrum demonstrates nonlinear effects when the pulse amplitude is large.

There is a significant quantitative difference between different xc potentials. In particular, we find that the excitonic features become more pronounced as one passes from KLI to Slater. The excitonic effects are defined mainly by the ratio of the Coulomb interaction energy to the typical energy of the free system. Since the valence and conduction bands and the gap have the same order of magnitude in our case, we can consider the ratio of the Coulomb energy to the valence bandwidth. In the Slater approximation, the amplitude of the Coulomb interaction energy appears to be bigger compared to KLI. In fact, as follows from the Appendix, the Coulomb interaction matrix elements are much larger in the Slater approximation, see Eqs. (A10), (A12), and (A13). In the strongly localized case considered in this paper, the Slater results are closer to HF (Fig. 5). For less localized electrons, when $a_{0} \sim 1$ and the momentum dependence of the xc potential matrix elements cannot be neglected, we expect to get closer agreement between Slater and KLI. Since KLI is a much better approximation for the exact OEP compared to the Slater potential, we expect the full XX-TDOEP absorption spectrum to be not too far from the KLI curve in Fig. 5. Another important conclusion from our numerical results is a decrease of the excitonic binding energy in Slater and KLI compared to HF. In fact, it is known that the HF approximation gives an overestimated binding energy of excitons (for a discussion, see Ref. 20).

Thus, we have shown that TDDFT can describe excitonic effects in the optical absorption spectra triggered by shorttime laser pulses, when the exchange energy kernel contains a long-range Coulomb singularity.

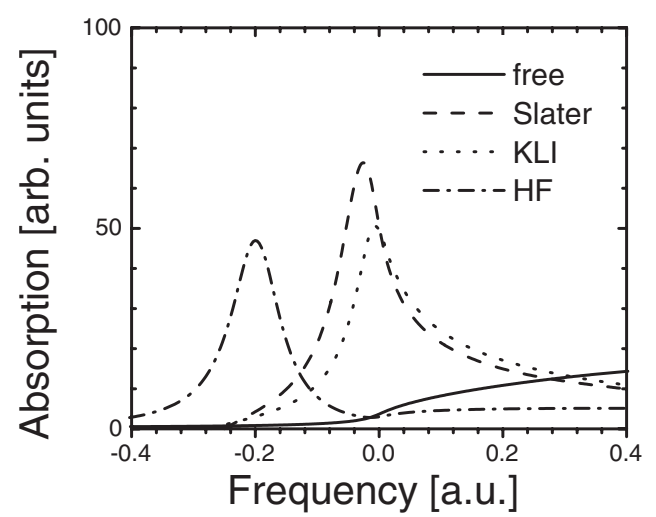

FIG. 5. Optical absorption spectra in different approximations at the same values of the model parameters. The valence bandwidth is equal to $0.05 \pi^{2}$ and the other parameters are given in the caption to Fig. 3. The Hartree-Fock result is obtained using a Bloch function approximation for the $\mathrm{xc}$ potential matrix elements (see the Appendix).

\section{CONCLUSIONS}

In this paper, a density-matrix TDDFT formalism to describe ultrafast processes in semiconductor structures has been developed. We have shown that the corresponding system of equations for the matrix elements is a generalization of the SBEs, since it allows one to take into account Coulomb effects, in principle, exactly. This is very important in the case of short pulses and when the system is very small, such as for nanostructures. Another important advantage of this formalism is that it can be applied directly in the time domain, which makes the calculation much faster.

As an example, we studied the optical absorption spectra of a two-band model bulk insulator. We first confirmed the well-known fact that the standard adiabatic LDA and GGA potentials do not produce excitonic peaks in the optical absorption spectra, not even if they have the correct asymptotic $-1 / r$ behavior for finite systems such as the LB potential. ${ }^{29}$ We then showed that the optical absorption spectra include excitonic effects when the xc energy kernel contains a $1 / q^{2}$ singularity. These findings are consistent with other TDDFT results for the optical response of insulators and semiconductors. ${ }^{14-16}$

In the TDDFT context, it is important to notice that these effects can be already described within an adiabatic approximation, i.e., without taking into account memory effects. The exciton binding energy in our model within the Slater and KLI approaches is much smaller compared to HF, which often gives too large values for the binding energy. However, to estimate the true quality of these approximate functionals, one needs to carry out more realistic calculations and compare with experimental absorption spectra and exciton binding energies. Such efforts are currently under way.

The density-matrix TDDFT approach presented in this paper should be useful for studying ultrafast optical processes in real semiconductor and polymer nanostructures. In particular, it gives access to a variety of nonlinear effects, which will be the subject of future studies.

\section{ACKNOWLEDGMENTS}

We thank Paul de Boeij for useful discussions and ac- 
knowledge financial support from NSF Grant No. DMR0553485 .

\section{APPENDIX: TECHNICAL DETAILS}

In this appendix, we present some useful approximations and technical details of the numerical solution of the TDDFT equations [Eqs. (19) and (20)]. Since, generally speaking, evaluation of the $\mathrm{xc}$ matrix elements beyond LDA requires six-dimensional spatial integration, it is necessary to make some simplifications to speed up the calculations. We shall use the Wannier and Bloch representation [Eqs. (23)-(27)] for the wave functions. In this case, the electron density can be expressed in the following form:

$$
n(\mathbf{r}, t)=2 \sum_{l, m, \mathbf{L}, \mathbf{q}} \rho_{\mathbf{q}}^{l m}(t) e^{-i \mathbf{L} q} w^{m^{*}}(\mathbf{r}-\mathbf{L}) w^{l}(\mathbf{r}) .
$$

Since the wave functions $w^{m}(\mathbf{r})$ and $w^{l}(\mathbf{r})$ are strongly localized, the main contribution to expression (A1) is given by the term $\mathbf{L}=0$. Therefore, the electron density is approximately

$$
n(\mathbf{r}, t) \simeq 2 \sum_{l, m} \rho_{t o t}^{l m}(t) w^{m^{*}}(\mathbf{r}) w^{l}(\mathbf{r}),
$$

where

$$
\rho_{t o t}^{l m}(t)=\sum_{\mathbf{q}} \rho_{\mathbf{q}}^{l m}(t)
$$

It turns out that Eq. (A2) can be obtained by another approximation for the wave functions, which follows from Eqs. (23)-(25). Namely,

$$
\begin{aligned}
& \psi_{\mathbf{k}}^{v}(\mathbf{r}) \simeq e^{i \mathbf{k r}} u_{\mathbf{k}_{0}}^{v}(\mathbf{r}), \\
& \psi_{\mathbf{k}}^{c}(\mathbf{r}) \simeq e^{i \mathbf{k r}} u_{\mathbf{k}_{0}}^{c}(\mathbf{r}) .
\end{aligned}
$$

We choose the wave vector $\mathbf{k}_{0}$ to be zero. This approximation is good when one considers excitation processes around the direct gap. In this case,

$$
\begin{aligned}
& \psi_{\mathbf{k}}^{v}(\mathbf{r}) \simeq e^{i \mathbf{k r}} w^{v}(\mathbf{r}), \\
& \psi_{\mathbf{k}}^{c}(\mathbf{r}) \simeq e^{i \mathbf{k r}} w^{c}(\mathbf{r}) .
\end{aligned}
$$

By using this approximation, one gets the following expression for the time-dependent matrix elements of the LDA and Slater potentials:

$$
\begin{gathered}
V_{L D A \mathbf{k}}^{l m}(t) \simeq-\left(\frac{3}{\pi}\right)^{1 / 3} \int_{\text {cell }} d \mathbf{r} w^{l^{*}}(\mathbf{r}) w^{m}(\mathbf{r}) \\
\times\left(\sum_{a, b} \rho_{\text {tot }}^{b a}(t) w^{a^{*}}(\mathbf{r}) w^{b}(\mathbf{r})\right)^{1 / 3}, \\
V_{\text {Slater } \mathbf{k}}^{l m}(t) \simeq-\sum_{n, s, \bar{n}, \bar{s}} \sum_{\mathbf{p}, \mathbf{q}} V(\mathbf{p}-\mathbf{q}) A^{l m n s \overline{n s}}(t) \rho_{\mathbf{p}}^{s n}(t) \rho_{\mathbf{q}}^{\overline{s n}}(t),
\end{gathered}
$$

where

$$
A^{l m n s \overline{n s}}(t) \simeq \int_{\text {cell }} d \mathbf{r} \frac{w^{l^{*}}(\mathbf{r}) w^{m}(\mathbf{r}) w^{n^{*}}(\mathbf{r}) w^{s}(\mathbf{r}) w^{\bar{n}^{*}}(\mathbf{r}) w^{\bar{s}}(\mathbf{r})}{n(\mathbf{r}, t)}
$$

and the density $n(\mathbf{r}, t)$ is given in Eq. (A2).

Finally, by using the Wannier wave functions in Eqs. (25)-(27) and making an approximation similar to that used in the derivation of Eqs. (A8) and (A9), one gets the following equation for the KLI matrix elements, which follows from Eq. (41):

$$
\begin{aligned}
V_{K L l \mathbf{k}}^{l m}(t)= & V_{\text {Slater } \mathbf{k}}^{l m}(t)+\sum_{n, s, \bar{n}, \bar{s}, \mathbf{p}} B_{1}^{l m n s}(t) \rho_{\mathbf{p}}^{s n} V_{K L l \mathbf{p}}^{\overline{n s}}(t) \rho_{\mathbf{p}}^{\overline{s n}} \\
& +\sum_{n, \bar{n}, s, \bar{s}, \mathbf{p}, \mathbf{q}} B_{2 \mathbf{p}}^{l m n s \bar{n}}(t) V(\mathbf{p}-\mathbf{q}) \rho_{\mathbf{q}}^{s n} \rho_{\mathbf{p}}^{\overline{s n}},
\end{aligned}
$$

where $\bar{V}_{\text {Slater }}(t)$ is defined in Eqs. (A9) and (A10), and

$$
\begin{gathered}
B_{1}^{l m n s}(t)=\int_{\text {cell }} d \mathbf{r} \frac{w^{l^{*}}(\mathbf{r}) w^{m}(\mathbf{r}) w^{n^{*}}(\mathbf{r}) w^{s}(\mathbf{r})}{n(\mathbf{r}, t)}, \\
B_{2 \mathbf{p}}^{l m n s \bar{n}}(t) \simeq-\int_{\text {cell }} d \mathbf{r} \frac{w^{l^{*}}(\mathbf{r}) w^{m}(\mathbf{r}) w^{n^{*}}(\mathbf{r}) w^{s}(\mathbf{r}) w^{\bar{n}^{*}}(\mathbf{r}) w^{\bar{s}}(\mathbf{r})}{n(\mathbf{r}, t)} \sum_{a, b} w^{a^{*}}(\mathbf{r}) w^{b}(\mathbf{r}) \rho_{\mathbf{p}}^{b a} \\
\simeq-\int_{\text {cell }} d \mathbf{r} \frac{w^{l^{*}}(\mathbf{r}) w^{m}(\mathbf{r}) w^{n^{*}}(\mathbf{r}) w^{s}(\mathbf{r}) w^{\bar{n}^{*}}(\mathbf{r}) w^{\bar{s}}(\mathbf{r}) w^{v^{*}}(\mathbf{r}) w^{v}(\mathbf{r})}{n(\mathbf{r}, t)} .
\end{gathered}
$$

It is convenient to solve Eq. (A11) by iteration. Therefore, we have reduced the six-dimensional space integral to a three-dimensional one. For numerical integration, we divide the space interval into 120 parts for every direction. In the case of momentum integration, we divide the interval into 100 parts. As it was mentioned in Sec. IV A, we use a decoherence factor $\Gamma$ in equations in order to reach a steady state faster. We have found that the position of the excitonic peak 
does not depend on value of $\Gamma$, but its height decreases with $\Gamma$ increasing. Also, it was found that in the Slater and KLI cases, the total absorption conservation law is satisfied with a higher precision when $\Gamma$ increases. Due to finite values of the time interval and time step, absorption $A(\omega)$ acquires unphysical finite weights at large values of $\left|\omega-\omega_{g}\right|$. These weights disappear when $\Gamma$ increases.

For completeness, let us present approximate matrix elements for the Hartree-Fock potential derived within the same approximation. These expressions are necessary to make a comparison of the absorption spectra using different potentials (Fig. 5). The Hartree-Fock matrix elements have the following structure:

$$
V_{H F \mathbf{k}}^{l m}(t) \simeq-\sum_{n, s} \sum_{\mathbf{p}, \mathbf{q}} V(\mathbf{k}-\mathbf{p}) A^{l m n s} \rho_{\mathbf{p}}^{s n}(t),
$$

where

$$
A^{l m n s} \simeq \int_{\text {cell }} d \mathbf{r} w^{l^{*}}(\mathbf{r}) w^{m}(\mathbf{r}) w^{n^{*}}(\mathbf{r}) w^{s}(\mathbf{r}) .
$$

Following the discussion in Sec. IV A, the quantities in Eq. (A15) can be approximated as $A^{l m n s} \simeq \delta^{l s} \delta^{n n}$. However, in approximations (A4) and (A5), these elements become

$$
A^{l m n s} \simeq \delta^{n n} I^{m} \int_{\text {cell }} d \mathbf{r} w^{l^{*}}(\mathbf{r}) w^{s}(\mathbf{r}) \simeq \delta^{n n} \delta^{l s} I^{m},
$$

where $I^{m}, m=v, c$, is the main contribution of the function $\left|w^{m}(\mathbf{r})\right|^{2}$ to the integral in Eq. (A15). Its value can be chosen to be equal to the maximum value of $\left|w^{m}(\mathbf{r})\right|^{2}$, i.e., $1 / \pi$ for the valence band function and $1 /\left(16 \pi e^{2}\right)$ for the conduction band function. In the last case, there is an additional factor $1 / 2$, which comes from the azimuthal angle integration. The factor $\pi / 6$ must be added in Eq. (A15), since in Slater and $\mathrm{KLI}$, there is an additional momentum integration over the Brillouin zone, and the integration is performed over a sphere of the radius $\pi$ instead of the cubic Brillouin zone.

The most important property of expressions (A8), (A9), and (A11) is the fact that they are momentum independent. Therefore, as follows from Eqs. (19) and (20), the matrix elements $\rho_{\mathbf{q}}^{m l}(t)$ are functions of the energy:

$$
\varepsilon_{\mathbf{k}}=\frac{1}{3}\left[\cos k_{x}+\cos k_{y}+\cos k_{z}\right] .
$$

In order to reduce the dimensionality of the integrals, it is convenient to introduce the corresponding three-dimensional density of states:

$$
\begin{aligned}
D(\varepsilon) & =\int \frac{d^{3} p}{(2 \pi)^{3}} \delta\left[\varepsilon-(1 / 3)\left(\cos q_{x}+\cos q_{y}+\cos q_{z}\right)\right] \\
& =\frac{3}{\pi^{3}} \int_{0}^{\pi} d p_{x} \int_{0}^{\pi} d p_{y} \frac{\theta\left[1-\left(3 \varepsilon-\cos p_{x}-\cos p_{y}\right)^{2}\right]}{\sqrt{1-\left(3 \varepsilon-\cos p_{x}-\cos p_{y}\right)^{2}}}
\end{aligned}
$$

where the energy $\varepsilon$ changes from -1 to 1 . The energy dependence of the density of states is shown in Fig. 6.

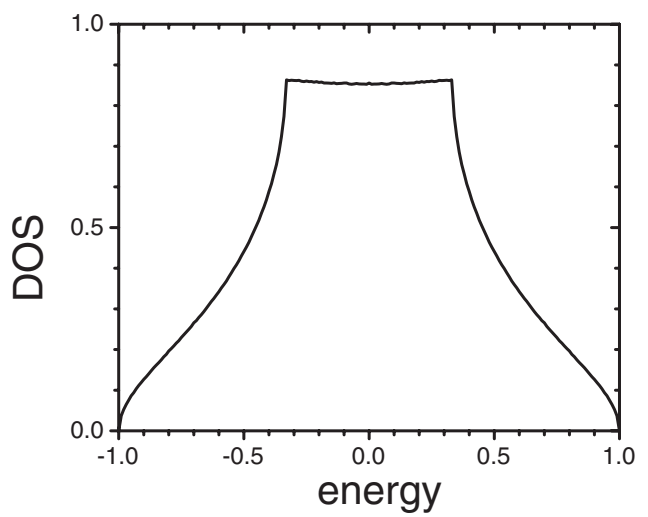

FIG. 6. The three-dimensional density of states [Eq. (A18)] as a function of energy.

Since the xc matrix elements depend on integrals which have the structure

$$
\int \frac{d^{3} p}{(2 \pi)^{3}} \int \frac{d^{3} q}{(2 \pi)^{3}} V(\mathbf{p}-\mathbf{q}) F\left(\rho_{\mathbf{p}}^{l m}, \rho_{\mathbf{q}}^{n s}\right),
$$

it is also convenient to introduce a two-energy density of states:

$$
\begin{aligned}
D_{2}(\varepsilon, \bar{\varepsilon})= & \int \frac{d^{3} p}{(2 \pi)^{3}} \int \frac{d^{3} q}{(2 \pi)^{3}} \delta\left[\varepsilon-(1 / 3)\left(\cos p_{x}+\cos p_{y}\right.\right. \\
& \left.\left.+\cos p_{z}\right)\right] \delta\left[\bar{\varepsilon}-(1 / 3)\left(\cos q_{x}+\cos q_{y}+\cos q_{z}\right)\right] V(\mathbf{p} \\
& -\mathbf{q}) .
\end{aligned}
$$

Performing the integration over $p_{z}$ and $q_{z}$ in Eq. (A20), one finds

$$
\begin{aligned}
D_{2}(\varepsilon, \bar{\varepsilon})= & \left(\frac{3}{\pi}\right)^{2} \int \frac{d^{2} p_{\|}}{(2 \pi)^{2}} \int \frac{d^{2} q_{\|}}{(2 \pi)^{2}} \frac{\theta\left[1-x^{2}\right]}{\sqrt{1+\delta-x^{2}}} \frac{\theta\left[1-y^{2}\right]}{\sqrt{1+\delta-y^{2}}} \\
& \times \frac{1}{\left(\mathbf{p}_{\|}-\mathbf{q}_{\|}\right)^{2}+\left(\cos ^{-1} x-\cos ^{-1} y\right)^{2}+1 / \lambda^{2}}, \quad(\mathrm{~A} 21)
\end{aligned}
$$

where $x=3 \varepsilon-\cos p_{x}-\cos p_{y}, y=3 \bar{\varepsilon}-\cos q_{x}-\cos q_{y}$, and $\mathbf{p}_{\|}$ $=\left(p_{x}, p_{y}, 0\right)$. In Eq. (A21), we have introduced "screening" parameters $\delta$ and $\lambda$, since in the three-dimensional case, the function $D_{2}(\varepsilon, \bar{\varepsilon})$ has a logarithmic singularity at $\varepsilon=\bar{\varepsilon}$. In fact, it is possible to show analytically that in the case of a square dispersion, $D_{2}(\varepsilon \rightarrow \bar{\varepsilon}) \sim \ln (1 /|\sqrt{\varepsilon}-\sqrt{\bar{\varepsilon}}|)$. We apply this approximation in the LDA case, see Fig. 2. In other figures, we use the parabolic band approximation for the sake of simplicity. This approximation gives results close to those obtained by using the densities of states [Eqs. (A18) and (A21)], when one uses a decoherence factor with a momentum cutoff (see Sec. IV A). In fact, in this case, low momenta give a dominant contribution to the absorption processes. 
*turkowskiv@missouri.edu

†ullrichc@missouri.edu

${ }^{1}$ G. Onida, L. Reining, and A. Rubio, Rev. Mod. Phys. 74, 601 (2002).

${ }^{2}$ F. Rossi and T. Kuhn, Rev. Mod. Phys. 74, 895 (2002).

${ }^{3}$ D. S. Chemla and J. Shah, Nature (London) 411, 549 (2001).

${ }^{4}$ H. Haug and S. W. Koch, Quantum Theory of the Optical and Electronic Properties of Semiconductors (World Scientific, Singapore, 2001).

${ }^{5}$ L. P. Kadanoff and G. Baym, Quantum Statistical Mechanics (Benjamin, New York, 1962).

${ }^{6}$ L. V. Keldysh, Zh. Eksp. Teor. Fiz. 47, 1515 (1964) [Sov. Phys JETP 20, 1018 (1965)].

${ }^{7}$ N. E. Dahlen and R. van Leeuwen, Phys. Rev. Lett. 98, 153004 (2007).

${ }^{8}$ W. Kohn, Rev. Mod. Phys. 71, 1253 (1999).

${ }^{9}$ E. Runge and E. K. U. Gross, Phys. Rev. Lett. 52, 997 (1984).

${ }^{10}$ E. K. U. Gross, J. F. Dobson, and M. Petersilka, in Density Functional Theory II, Topics in Current Chemistry Vol. 181 (Springer, New York, 1996), p. 81.

${ }^{11}$ Time-Dependent Density Functional Theory, edited by M. A. L. Marques, C. A. Ullrich, F. Nogueira, A. Rubio, K. Burke, and E. K. U. Gross, Lecture Notes in Physics Vol. 706 (Springer, Berlin, 2006).

${ }^{12}$ P. Elliott, K. Burke, and F. Furche, arXiv:cond-mat/0703590 (unpublished).

${ }^{13}$ S. Botti, A. Schindlmayr, R. Del Sole, and L. Reining, Rep. Prog. Phys. 70, 357 (2007).

${ }^{14}$ L. Reining, V. Olevano, A. Rubio, and G. Onida, Phys. Rev. Lett. 88, 066404 (2002).

${ }^{15}$ A. Marini, R. Del Sole, and A. Rubio, Phys. Rev. Lett. 91,
$256402(2003)$

${ }^{16} \mathrm{~S}$. Botti, F. Sottile, N. Vast, V. Olevano, L. Reining, H.-C. Weissker, A. Rubio, G. Onida, R. Del Sole, and R. W. Godby, Phys. Rev. B 69, 155112 (2004).

${ }^{17}$ Y. H. Kim and A. Görling, Phys. Rev. Lett. 89, 096402 (2002).

${ }^{18}$ Y. H. Kim and A. Görling, Phys. Rev. B 66, 035114 (2002).

${ }^{19}$ H. O. Wijewardane and C. A. Ullrich, Phys. Rev. Lett. 100, 056404 (2008).

${ }^{20}$ F. Bruneval, F. Sottile, V. Olevano, and L. Reining, J. Chem. Phys. 124, 144113 (2006).

${ }^{21}$ W. Schäfer and M. Wegener, Semiconductor Optics and Transport Phenomena (Springer, Berlin, 2002).

${ }^{22}$ N. T. Maitra, I. Souza, and K. Burke, Phys. Rev. B 68, 045109 (2003).

${ }^{23}$ M. van Faassen, Int. J. Mod. Phys. B 20, 3419 (2006).

${ }^{24}$ H. O. Wijewardane and C. A. Ullrich, Appl. Phys. Lett. 84, 3984 (2004).

${ }^{25}$ K. Burke, R. Car, and R. Gebauer, Phys. Rev. Lett. 94, 146803 (2005).

${ }^{26}$ O. Berman and S. Mukamel, Phys. Rev. A 67, 042503 (2003).

${ }^{27}$ A. V. Maslov and D. S. Citrin, Phys. Rev. B 62, 16686 (2000).

${ }^{28}$ A. V. Maslov and D. S. Citrin, Phys. Rev. B 64, 155309 (2001).

${ }^{29}$ R. van Leeuwen and E. J. Baerends, Phys. Rev. A 49, 2421 (1994).

${ }^{30}$ J. P. Perdew and A. Zunger, Phys. Rev. B 23, 5048 (1981).

${ }^{31}$ C. A. Ullrich, P.-G. Reinhard, and E. Suraud, Phys. Rev. A 62, $053202(2000)$

${ }^{32}$ C. A. Ullrich, U. J. Gossmann, and E. K. U. Gross, Phys. Rev. Lett. 74, 872 (1995).

${ }^{33}$ J. B. Krieger, Y. Li, and G. J. Iafrate, Phys. Rev. A 45, 101 (1992). 